Trab. Ling. Aplic., Campinas, 44(1): 37-58, Jan./Jun. 2005

\title{
LA FRASEOLOGÍA: ENTRE EL LENGUAJE REAL Y LA CLASE DE E/LE ${ }^{1}$
}

\author{
LÍVIA MÁRCIA TIBA RÁDIS BAPTISTA - Posgrado FE/USP \\ MARÍLIA OLIVEIRA VASQUES CALLEGARI - Posgrado FE/USP \\ MARIA ETA VIEIRA - Posgraduada en Historia FFLCH/USP \\ SIMONE RINALDI - Posgrado FE/USP \\ I. GRETEL M. ERES FERNÁNDEZ - Doctora en Educación FE/USP²
}

\begin{abstract}
RESUMO
Este trabalho tem por finalidade refletir acerca do ensino e da aprendizagem do léxico, em especial, das unidades fraseológicas. Sendo assim, propõe demonstrar a importância de proporcionar aos aprendizes do espanhol como língua estrangeira o conhecimento e a habilidade no emprego das unidades fraseológicas, mais exatamente, das expressões idiomáticas. Inicialmente expõe um quadro teórico, no interior do qual examina questões como, por exemplo, a problemática em torno de uma definição conceitual das unidades fraseológicas ou formas fixas, a necessidade e relevância da seleção léxica nos âmbitos comunicativo e pedagógico, a relevância das expressões idiomáticas como objeto de ensino e da aprendizagem e, ainda, os modos de inseri-las na aula. Em seguida, analisa de que modo são apresentadas as expressões idiomáticas, a partir da tipologia de exercícios e de atividades propostas em seis manuais de espanhol. Como critérios para definição do corpus, considerou-se o fato de serem manuais de difusão ampla e generalizada no Brasil, seja no ensino fundamental, seja nos cursos livres de idiomas. Além disso, optou-se por manuais publicados tanto na Espanha como no Brasil, ao longo dos anos 90, orientados por diversas propostas metodológicas e classificados, conforme os seus autores, nos níveis básico a avançado. Com base na análise, inferiu-se que as expressões idiomáticas predominam nos níveis considerados mais avançados do idioma e que parece não haver uma grande preocupação no sentido de, efetivamente, estimular, nos aprendizes, a ampliação do uso das expressões idiomáticas a outros contextos nos quais essas possam fazer sentido.
\end{abstract}

Palavras-chave: léxico; expressão idiomática; ensino; aprendizagem.

\begin{abstract}
The purpose of this study is to reflect on the teaching and learning of lexis, in particular, phraseological units. We aim to show the importance of providing learners of Spanish as a Foreign Language with the knowledge and skills in using phraseological units, more specifically, idiomatic expressions. Starting from a theoretical base, we examine questions such as the problems regarding the conceptual definition of phraseological units or fixed forms, the need and relevance of lexical selection in the communicative and pedagogical ambit, the relevance of idiomatic expressions as an object of teaching and learning, as well as how to incorporate them in the classroom. Next we examine the types of exercises and activities proposed in six Spanish coursebooks in order to analyze
\end{abstract}

\footnotetext{
${ }^{1}$ Este trabajo constituye una ampliación teórica de la ponencia "Vamos al grano y salgamos de apuros: ¡las expresiones idiomáticas no son un lío”, presentada en el IX Seminario de Dificultades Específicas en la Enseñanza del Español a Lusohablantes. São Paulo, septiembre/02.

${ }^{2}$ Participaron en la primera etapa de este trabajo, además de las profesionales indicadas, las profesoras Eliane Gonçalves - Posgraduada en Letras FFLCH/USP y Magali de Lourdes Pedro - Posgrado FFLCH/USP.
} 
how idiomatic expressions are presented. When defining the corpus, the fact that the coursebooks are widely used, both in secondary schools and in the private language schools, was taken into consideration. In addition, we chose coursebooks with differing methodological approaches published both in Spain and Brazil during the nineties, and classified them from basic to advanced level. Based on the analysis, we inferred that idiomatic expressions predominate in the more advanced levels, and that there seems to be little preoccupation with stimulating the learners to use the expressions more widely in other meaningful contexts.

Key-words: lexis; idiomatic expressions; teaching; learning.

\section{INTRODUCCIÓN}

La enseñanza y el aprendizaje del léxico constituyen uno de los principales retos con el que a menudo nos enfrentamos en una clase de lenguas que se considere comunicativa, o aun, basada en un abordaje comunicativo. Prueba de ello es la necesidad real que tenemos de mostrar y de ofrecer a los alumnos un vocabulario mínimo con el que puedan manejarse en diferentes contextos comunicativos. De ahí, la necesidad de que los aprendices sean competentes, comunicativamente, en la lengua extranjera se asocie al uso de la lengua y no solamente a su conocimiento sistémico. Dada la amplitud del asunto, a continuación, recordaremos brevemente el concepto de competencia comunicativa, teniendo en cuenta lo propuesto por Dell Hymes en los años 60 y ampliado, posteriormente, por Canale (Llobera, 1995:5-23).

Para Dell Hymes, la competencia comunicativa presenta cuatro dimensiones: la competencia lingüística, la factibilidad, la aceptabilidad o adecuación y la realidad. De ese modo, la competencia lingüística abarca las reglas gramaticales, las cuales se refieren a la actuación lingüística. La factibilidad se refiere, entre otros, al hecho de que se puede utilizar las expresiones de acuerdo con los medios disponibles ${ }^{3}$ : fatiga, formación, edad, etc. $\mathrm{La}$ aceptabilidad o adecuación se refiere al hecho de que la actuación lingüística se realiza bajo determinadas reglas sociolingüísticas del contexto en el que se produce la comunicación. El último aspecto, el de la realidad, se refiere al hecho de que el hablante no puede inventarse usos de la lengua. Los puntos señalados, en su mayoría, se dan, a la hora de comunicarse, de manera inconsciente.

Michel Canale, a su vez, aplica el concepto de competencia comunicativa a la didáctica. Su modelo es más amplio y menos programático que el modelo de Dell Hymes, como nota Llobera (1995:14). Desde esta perspectiva, la competencia comunicativa se divide en las siguientes subcompetencias: la gramatical, la sociolingüística, la discursiva y la estratégica. Cabe añadir que Llobera (1995:14-25) analiza cada una de esas y su desarrollo en el aula. Proponemos comentar, a partir de lo propuesto por Llobera, las mencionadas subcompetencias que juntas constituyen la macro competencia comunicativa.

\footnotetext{
${ }^{3}$ Aunque Dell Hymes no aclare totalmente qué se debe entender por “medios disponibles”, interpretamos que entre ellos estarían los que pueden - en alguna medida - limitar la actuación lingüística. De ahí, que la referencia al estado anímico o físico, la edad o la formación se justifiquen.
} 
La subcompetencia gramatical se refiere al dominio del código lingüístico, sea verbal o no verbal, o aun, al dominio de determinadas reglas estructurales de la lengua, que, a su vez, se relacionan con sus niveles fonológico, sintáctico, semántico y lexical. La competencia sociolingüística se refiere a las reglas socioculturales de uso y de discurso de la lengua, pues esa es el medio de comunicación entre los hablantes que en diferentes contextos sociolingüísticos y conforme los factores contextuales producen e interpretan las expresiones lingüísticas. La subcompetencia discursiva se relaciona con la manera como se unen las formas gramaticales y los significados para componer un texto, es decir, se refiere a los aspectos de cohesión y coherencia responsables no sólo de la organización sino también de la interpretación del texto o textos. Y, por fin, la subcompetencia estratégica se relaciona con la habilidad de los hablantes para utilizar estrategias verbales o no verbales con la finalidad de compensar fallos en la comunicación o mejorar su actuación en ella.

Teniendo en cuenta lo antedicho, nos detendremos en el nivel lexical, entre otras razones porque el léxico no sólo integra la subcompetencia gramatical, sino que también se relaciona con el conocimiento de las reglas de uso, es decir, integra la subcompetencia sociolingüística y aún se asocia sea con la cohesión, sea con la coherencia y, por lo tanto, a la subcompetencia discursiva.

A partir de lo expuesto, inferimos que el dominio del código lingüístico no puede prescindir del conocimiento y uso de las reglas de adecuación del significado al contexto comunicativo y de los elementos de cohesión y de coherencia del discurso. De ese modo, la falta de conocimiento y de habilidad en el empleo, por parte del aprendiz, de algunas reglas socioculturales de uso, que orientan la producción e interpretación del enunciado, puede engendrar malentendidos, ruptura en la comunicación o irritación en el oyente. De esa forma, el conocimiento y la habilidad para utilizar la lengua, de modo a explotar sus matices expresivos y significativos, adecuar los sentidos a las distintas situaciones y contextos, forman parte de la realidad lingüística. Y si forman parte de dicha realidad y si pretendemos el desarrollo de la mencionada competencia comunicativa en determinada lengua, deben ser objeto de la enseñanza y del aprendizaje.

Con base en lo mencionado, notamos que hay, en gran parte de los diseños de curso y de los libros de texto, una significativa valoración del conocimiento sistémico y que, muchas veces, no se reconoce como de igual importancia el conocimiento del léxico para obtener la competencia comunicativa en la lengua meta y tampoco su relación con las demás subcompetencias como señalamos. No se trata de ignorar o negar o menospreciar en el ámbito de la enseñanza y del aprendizaje la importancia de los aspectos fonológicos, sintácticos y morfológicos para lograr el conocimiento de la organización discursiva y textual, sino de reconocer y facilitar el uso y la asimilación de determinados elementos del léxico, es decir, de potenciar su aprendizaje para promocionar la mencionada competencia. Conviene notar que aunque en el aula el profesor se preocupe o se interese por el léxico y por los aspectos semánticos, en muchos momentos no logra relacionarlos a las demás competencias, en especial, a la sociolingüística y a la discursiva.

Teniendo en cuenta las aclaraciones anteriores, ¿qué sería posible enseñar en términos de léxico de una lengua? Desde la perspectiva de la enseñanza y del aprendizaje, que es la 
que orienta este trabajo, reconocemos que hay que operar con recortes que, a su vez, faciliten sea la organización de contenidos, sea la definición de objetivos, sea la sugerencia de propuestas didácticas. En este sentido, enfocaremos una parte significativa del léxico y que integra la fraseología. Por razones de delimitación, en este estudio nos ocuparemos, en especial, de determinados aspectos que estimamos relevantes para potenciar la enseñanza y el aprendizaje del léxico ${ }^{4}$, más exactamente, de las expresiones idiomáticas.

Con el propósito de facilitar la lectura y comprensión de nuestros análisis, en los próximos apartados examinaremos los siguientes puntos: en qué consiste la selección de las expresiones y cuál es su necesidad para la enseñanza, cómo introducirlas en el aula y cuáles son las actividades que pueden llevar a una práctica más efectiva y a su fijación. Además, analizaremos algunos materiales didácticos de español con la finalidad de averiguar cómo figuran las expresiones idiomáticas en ellos y cuáles son los tipos de actividades propuestas.

\section{LA SELECCIÓN Y EL MODO DE ENSEÑANZA DE LAS UNIDADES FRASEOLÓGICAS}

Debido a la amplitud y a la complejidad del tema en sí, limitaremos nuestras reflexiones a dos cuestiones específicas a partir de las cuales profundizaremos nuestras consideraciones posteriores: a) la selección del léxico y b) el modo de enseñarlo. En este sentido, comentaremos en qué consiste tal selección y su importancia. Con fines teóricos y a la vez didácticos solamente nos ocuparemos de las expresiones idiomáticas en este estudio, aunque reconozcamos que forman parte de la enseñanza del léxico en sentido amplio. Cabe señalar, todavía, que las expresiones idiomáticas se incluyen en lo que Penadés (1999) considera unidades fraseológicas y, así, posteriormente, veremos en qué consisten esas y cómo se pueden caracterizar las expresiones idiomáticas. A continuación, examinaremos cómo proceder la selección del léxico y cómo enseñarlo.

Con respecto a la selección del léxico, desde la perspectiva de la metodología de enseñanza de lenguas extranjeras, que es la que nos atañe, hay que tener presentes los siguientes puntos:

a) cuáles son las palabras que debemos o podemos elegir para enseñar;

b) cuáles son los criterios o métodos que podemos seguir en dicha elección.

Además de lo anterior, en cuanto al modo de enseñar el léxico se debe tener en cuenta cuál es el interés e importancia de las unidades fraseológicas - entre ellas de las expresiones idiomáticas - para los aprendices brasileños de español como lengua extranjera.

\footnotetext{
${ }^{4}$ Este trabajo ha sido desarrollado por el "Grupo de Pesquisa Ensino-Aprendizagem de Espanhol - USP/ CNPq" y tiene como principal objetivo discutir y reflexionar sobre la importancia y necesidad de incluir en la programación de cursos de LE el estudio de las expresiones idiomáticas.
} 
Por lo tanto, nos ocuparemos de los aspectos mencionados, de modo bastante general, con la finalidad de reflexionar acerca de las dificultades presentes sea en la enseñanza, sea en el aprendizaje del léxico. Sin embargo, reconocemos que aunque nuestro foco sean las expresiones idiomáticas, las reflexiones que incluimos a continuación podrán contribuir a que se realicen otros estudios y propuestas de aplicación didáctica que contemplen distintos elementos del léxico.

\section{NECESIDAD DE SELECCIÓN DE LAS EXPRESIONES IDIOMÁTICAS PARA LA ENSEÑANZA}

La selección y la adecuada utilización del léxico forman parte de las subcompetencias gramatical y sociolingüística que, a su vez, integran la competencia comunicativa. Por ello, es necesario seleccionar el vocabulario en función de las necesidades e intenciones adecuadas a cada contexto comunicativo, hecho que se verifica tanto en el ámbito de la enseñanza y aprendizaje como en el ámbito de la comunicación. A partir de esa premisa, podemos afirmar que la selección del léxico forma parte, también, de la competencia estratégica en la medida en que constituye un recurso del que el hablante echa mano para mejorar su actuación en la lengua. Así, comentaremos, a seguir, algunos métodos y criterios propuestos para la selección del léxico a partir de De Grève (apud Passel, 1983) y Halliday et alii (1974).

De Gréve (apud Passel, 1983) propone para la selección del léxico tres métodos: los de selección subjetiva o empírica, los de selección estrictamente objetiva y los de objetividad dirigida.

En el método de la selección subjetiva mencionado por De Grève, se eligen al azar y arbitrariamente los términos lexicales que se deben enseñar sin tener en cuenta las necesidades comunicativas o el nexo semántico. La principal crítica a tal método es su falta de rigor científico.

La selección estrictamente objetiva se establece con base en la ocurrencia de la palabra, es decir, enfatiza su valor frecuentativo. De Grève (apud Passel, 1983) propone que se haga la selección del léxico con base en la lengua escrita, que considera superior a la oral, aunque este criterio puede generar distorsiones entre la frecuencia absoluta y la relativa. En un determinado género de texto, por ejemplo, pueden aparecer términos relacionados con las regiones polares, en que serán frecuentes los términos "iglú, reno, trineo", sin que por este motivo sean términos de ocurrencia absoluta en la lengua.

Por fin, la selección con objetividad dirigida abarca dos principios: el de las "cualidades generativas de palabra" y el de la "disponibilidad". El primer principio considera fundamental, para el reconocimiento y la asimilación de la palabra, su aproximación a una "raíz" ya asimilada por el aprendiz. El segundo principio considera los sustantivos elementos fundamentales para la selección.

Además de lo ya mencionado, tenemos los criterios frecuentativo y comunicativo propuestos por Halliday et alii (1974). El primero de ellos establece listas de frecuencia de 
palabras y de expresiones de acuerdo con su ocurrencia en la lengua; ya el segundo propone listas de nociones y funciones que se vuelcan hacia la importancia que tienen para una dada situación comunicativa. De todos modos, la selección se apoya en listas de uso de determinados términos por dos motivos: su frecuencia en la lengua, de modo absoluto, sin contemplar contextos de uso y su aparición en dichos contextos, a través de una lista de algunas nociones y funciones que se repiten.

Los métodos y criterios referidos, aunque puedan orientar la selección del léxico, no son totalmente satisfactorios para potenciar el uso de las expresiones idiomáticas y, diríamos, de las demás unidades fraseológicas. Y en tal caso, una dificultad se plantea, es decir, si hace falta escoger o seleccionar, o aun, organizar el input ${ }^{5}$ lexical que se va a ofrecer a los aprendices, ¿cuáles son las palabras que debemos o podemos enseñar a nuestros alumnos? Bartol (apud Slagter,1988) contesta a esa pregunta proponiendo que les enseñemos a los alumnos un "léxico fundamental" y que el profesor facilite el acceso a los "medios que permitan al estudiante la comprensión de un número mayor de palabras". Es decir, que se enseñe un vocabulario básico que le sirva al aprendiz para establecer comunicación, comprensión y participación en distintas situaciones comunicativas.

En cuanto a la elección de las palabras que podemos y debemos enseñar conviene considerar los siguientes aspectos: los intereses y el nivel de conocimiento lingüístico de los alumnos, los distintos objetivos de los cursos, y aun, las diferencias del léxico en cuanto a registro, usos, valores, matices de sentido, etc. que puedan representar dificultad para el aprendiz de determinado nivel. La enseñanza de neologismos, arcaísmos o dialectos es necesaria y útil cuando atiende a los intereses y objetivos ya señalados. Resulta productiva también la enseñanza de otros términos, como palabrotas, jergas, expresiones idiomáticas, coloquialismos o muletillas en la medida en que permiten una mejor actuación en la lengua a nivel pragmático-discursivo. La enseñanza de términos específicos del mundo del comercio, del turismo, de los negocios, por ejemplo, interesa también a aquellos aprendices que trabajan directamente con dicho universo y el conocimiento de tales términos tiene una finalidad comunicativa real al paso que la falta de ese conocimiento puede engendrar problemas en la vida práctica. Por lo tanto, la selección del léxico y de las expresiones idiomáticas en concreto debe pautarse por los aspectos mencionados, es decir, debe atender al perfil del grupo, a necesidades comunicativas y discursivas, y aun, orientarse hacia la dificultad que puedan representar para el aprendiz en términos de comprensión de su uso y asimilación a otros contextos.

\section{MODOS DE ENSEÑANZA DE LAS UNIDADES FRASEOLÓGICAS}

Otro aspecto que interesa tratar es el de los modos de enseñar el léxico. Aclaramos que desde la perspectiva metodológica enfatizaremos las unidades fraseológicas que, a

\footnotetext{
${ }^{5}$ Se entiende por input, en este trabajo, la muestra de lengua ofrecida a los alumnos.
} 
su vez, incluyen las expresiones idiomáticas. Justificamos esta opción porque, muchas veces, nuestros alumnos nos preguntan acerca de ellas pues no entienden su sentido o sentidos lo cual puede representar una dificultad para la comprensión adecuada de todo el mensaje. Como hemos notado, por parte de aprendices brasileños, la preocupación y la duda con respecto a lo que significan y cuándo y cómo utilizamos las unidades fraseológicas de manera adecuada y significativa, consideramos necesario examinar algunas cuestiones:

a) ¿Qué es una unidad fraseológica?

b) ¿Cómo se definen o qué caracteriza las expresiones idiomáticas?

c) ¿Cuál es el interés e importancia de las expresiones idiomáticas para el aprendizaje y la enseñanza del español a brasileños?

Sin embargo, reconocemos que, dadas las limitaciones de este trabajo, estudios más profundos sobre el tema se hacen necesarios. Nuestro objetivo, en este momento, es el de introducir el léxico en el foco de la reflexión acerca de la enseñanza y del aprendizaje de español, y más específicamente, las expresiones idiomáticas.

En cuanto a la pregunta inicial, ¿qué es una unidad fraseológica? Penadés Martínez (1999:12-14) observa que el término unidad fraseológica o fraseologismo abarca tanto lo que tradicionalmente denominamos dichos, expresiones fijas, expresiones idiomáticas, expresiones sin más, frases, modismos, giros, fórmulas, y también fórmulas proverbiales ofórmulas comunicativas, idiotismos, locuciones, modos de decir, frases hechas, refranes, adagios, proverbios o aforismos, como lo que hoy día llamamos colocaciones, expresiones o unidades pluriverbales, lexicalizadas o habitualizadas y unidades léxicas pluriverbales. La autora señala que el término "unidades fraseológicas" es genérico, pero es el que se impone cada vez más; sin embargo, eso no significa que no haya diferencias entre un tipo y/u otro, por ejemplo, entre una "locución" y un "refrán".

Con respecto a las expresiones idiomáticas, una forma fija o una unidad fraseológica, resumiremos sus características a partir de lo propuesto por Zuluaga (1980), quien considera que:

1) son parte del saber lingüístico de la comunidad.

2) están institucionalizadas, estandarizadas y convencionalizadas.

3) son fijadas arbitrariamente por el uso repetido en la comunidad lingüística respectiva, es decir, son reproducidas en el habla como construcciones previamente hechas.

4) las define alguna forma de fijación, es decir, en las unidades fraseológicas está suspendida alguna regla de combinación de los elementos del discurso.

5) se destacan por su estructura material, por su iconicidad y por sus rasgos semánticos peculiares, por comentarios metalingüísticos y por emplearse en forma recortada. 
Además, Zuluaga (1980) señala que:

1) a pesar de la fijación - su rasgo definitorio -, las formas fijas, entre las cuales están las expresiones idiomáticas ${ }^{6}$ se emplean alteradas o modificadas en su estructura interna es decir, en el inventario de sus componentes o en el orden, la cohesión, las categorías gramaticales de los mismos - o en su combinalidad con otros elementos del discurso.

2) son construcciones cortas.

3) dan un alto relieve al mensaje, al texto o al segmento de texto en que se empleen.

4) presentan un contenido mediante una imagen concreta de orden visual. Tienen, pues, un sentido literal - la imagen - y un sentido metafórico - idiomático o semiidiomático.

¿Cuál es el interés e importancia de las expresiones idiomáticas para el aprendizaje y la enseñanza del español a brasileños? Ya tratamos, en alguna medida, esta cuestión al principio de este estudio cuando enfocamos la importancia de la enseñanza del léxico y de su relación con la competencia comunicativa. Además, conviene insistir en que el conocimiento y el empleo adecuado de las expresiones idiomáticas implica el dominio y habilidad de uso de ciertas reglas de organización y adecuación discursiva y pragmática de la lengua. Por otra parte, al conocer y al emplear las expresiones idiomáticas el aprendiz logrará interpretar cómo cambian los sentidos, no se retringirá a una comprensión literal, muchas veces equivocada, de determinados términos.

Sin embargo, sabemos que la enseñanza de las unidades fraseológicas - en especial de las expresiones idiomáticas - le resulta al profesor de español como lengua extranjera bastante compleja por la carencia de investigaciones que le puedan indicar qué unidades fraseológicas debe enseñar en cada nivel, por la raridad de materiales específicos que den soporte a su labor y por la falta de adecuación de los recursos didácticos, conforme nota Penadés Martínez (1999:22). Hay que añadir, según la misma autora (1999:22), la dificultad de la fijación formal y la idiomaticidad de las unidades fraseológicas.

A partir de las afirmaciones anteriores, Penadés Martínez (1999:24) llama la atención hacia la necesidad de más investigaciones sobre las unidades fraseológicas que nos puedan mostrar, al menos, el registro al que pertenecen, su frecuencia y sus condiciones de uso. A pesar de que existen algunos diccionarios y repertorios fraseológicos, también existe carencia de estudios teóricos sobre la fraseología española y faltan trabajos descriptivos acerca de las unidades fraseológicas.

Aún de acuerdo con Penadés Martínez (1999:24-35), el profesor dispone de dos materiales, específicamente, para la enseñanza de las unidades fraseológicas: libros de

${ }^{6}$ Esclarecemos que Penadés Martínez (1999) se basa en el mencionado trabajo de Zuluaga (1980). La autora (1999:15-17) para definir en qué consisten las unidades fraseológicas recuerda que Zuluaga considera como elementos definidores de las expresiones/formas fijas la fijación y la idiomaticidad. Por lo tanto, deducimos que el término unidades fraseológicas equivale, aquí, a expresiones/formas fijas de Zuluaga. Además de eso, conforme esa autora (1999:11), las unidades fraseológicas son "combinaciones de palabras que muestran un alto grado de fijación en su forma y en su significado" y pueden corresponder a diversos niveles lingüísticos. Por lo tanto, las expresiones idiomáticas serían formas/expresiones fijas y/o unidades fraseológicas. 
texto y diccionarios. Aunque no sea nuestro objetivo, en este estudio, analizar dichos materiales, vale señalar que presentan algunas deficiencias, en especial los diccionarios, en lo que se refiere a la selección y ordenación de las unidades fraseológicas por niveles. Señalamos, además, que analizamos algu y superior producidos en la década de los 90 y constatamos que no había un interés especial por las expresiones idiomáticas, jergas, muletillas, coloquialismos, etc. Notamos que tales aspectos sólo aparecen - cuando aparecen - en manuales de lengua de nivel avanzado o superior. Probablemente eso se deba al hecho de que se consideran sofisticadas esas cuestiones para el aprendiz de la lengua extranjera. Sin embargo, el profesor no puede ignorarlas en la lengua en uso o dejar de considerarlas como fundamentales para que los aprendices alcancen la competencia comunicativa en la lengua meta, conforme demostramos aquí.

Considerándose lo expuesto, la autora (1999) propone lo que denomina una "didáctica de las unidades fraseológicas". Resumiremos, a continuación, algunos puntos que estimamos de relieve y que podrán orientar la enseñanza de las expresiones idiomáticas a aprendices brasileños de español como lengua extranjera.

Penadés Martínez (1999:35) menciona la propuesta de Vigara Tauste (1996:86-93) de presentar las unidades fraseológicas a partir de un ejemplo-base y de ofrecer listas de unidades fraseológicas elaboradas desde algún criterio unificador que puede ser un área temática (como el color, las relaciones familiares o las partes del cuerpo). A título ilustrativo, señala las locuciones en las que figura la palabra "cabeza": "tener mala cabeza, romperse la cabeza, calentarle a alguien la cabeza", etc. Además, Penadés Martínez (1999:36) menciona la propuesta de Sevilla Muñoz y González Rodríguez (1994-95:172-173) para quienes el profesor debe:

1) explicar el sentido y origen de la expresión o unidad fraseológica, si posible;

2) enseñarlas aisladas o en su contexto;

3) proporcionar unidades fraseológicas sinónimas y antónimas;

4) señalar su registro y su frecuencia.

De lo anterior se deducen dos momentos importantes: 1) el de la presentación a los alumnos del contenido (expresiones idiomáticas, por ejemplo) y 2) el de la práctica. Tras la presentación, se pasa a la práctica, que comprende tres momentos distintos: a) la comprobación del significado y del uso del contenido previamente presentado; b) la transferencia de ese contenido a otros contextos; y c) la memorización y refuerzo del contenido. Trataremos, a continuación, de explicitar algunas propuestas para esos momentos.

Penadés Martínez (1999:36-43) propone algunos ejercicios para la presentación y para la práctica (reutilización y memorización de las unidades fraseológicas) y presenta algunos ejemplos.

Para la presentación, la autora se apoya en los pasos propuestos por Sevilla Muñoz y González Rodríguez (1994-95:172-173) ya mencionados.

En un segundo momento, es decir, después de la fase de presentación, propone la práctica con la finalidad de comprobar si el alumno ha captado o no el significado y el uso 
de las unidades fraseológicas, si puede volver a emplearlas en otras ocasiones y en otras prácticas y si las ha memorizado. Para averiguar si el alumno ha captado el significado y el uso de las unidades fraseológicas, el profesor puede proponer a los aprendices algunas actividades:

a) relacionar la unidad fraseológica con su significado

b) comparar unidades fraseológicas de la lengua materna con las de la lengua que está aprendiendo

c) redactar un texto nuevo en el cual haya unidades fraseológicas que deberá sustituir por su significado.

d) señalar las diversas acepciones de una unidad fraseológica homónima, con énfasis en su significado literal y en su idiomaticidad.

El tercer paso y con el objetivo de que el alumno utilice las unidades fraseológicas y las expresiones idiomáticas - conocidas, el profesor puede solicitarle que:

a) complete un texto con una unidad fraseológica seleccionada de un conjunto de tres o cuatro opciones

b) añada una unidad fraseológica en huecos de textos ya trabajados en clase

c) busque distintos contextos en los que pueda insertar una unidad fraseológica homónima

d) utilice las unidades fraseológicas en dramatizaciones, diálogos de libre creación o descripciones acerca de temas cotidianos con el objetivo de averiguar si es capaz de emplear las unidades vistas de forma oral con alguna soltura.

Por último, con el propósito de memorizar estas unidades, el profesor puede solicitar que el alumno:

a) busque, a partir de una unidad fraseológica presente en un texto, otras similares, o sea, que pertenezcan al mismo campo semántico y la correspondencia en la lengua materna parte

b) complete expresiones fijas de las cuales el profesor solamente le ofrece la primera

c) reconstruya las formas originales de ciertos fraseologismos ya conocidos a partir de su mezcla

d) señale qué unidades fraseológicas se confunden en una especie de "sopa de letras" que incluye los elementos de diferentes unidades fraseológicas.

\section{LA FRASEOLOGÍA Y LOS LIBROS DE TEXTO}

En los apartados anteriores hemos discutido la importancia de la fraseología y las dificultades que suponen tanto su conceptuación como su clasificación. Hemos presentado, 46 
también, algunas propuestas prácticas de trabajo para la clase de E/LE a partir de la selección de algunas unidades fraseológicas. A continuación discutiremos brevemente cómo algunos materiales didácticos de español para extranjeros tratan el asunto.

A lo largo de nuestra experiencia docente hemos tenido que hacer frente a preguntas de alumnos sobre el significado de determinadas expresiones idiomáticas presentes en libros de texto y a la dificultad que muchos tenían de transformar ese conocimiento en vocabulario activo. En otras ocasiones se eludía el problema puesto que el libro de texto utilizado apenas incluía expresiones idiomáticas. Entre una situación y otra, y si se considera que el libro es un recurso muy utilizado por gran parte del profesorado brasileño, se justifica un breve análisis cuantitativo y calitativo de algunos de esos materiales en lo que a las expresiones idiomáticas se refiere.

Procedimos, pues, a la compilación de las expresiones idiomáticas presentes en seis libros de texto de E/LE, elegidos al $\mathrm{azar}^{7}$ y de distintos niveles (básico, intermedio, avanzado y superior) y llegamos al siguiente cuadro:

\begin{tabular}{|l|l|}
\hline LIBRO & TOTAL DE EXPRESIONES \\
\hline Español sin Fronteras (Scipione -4 tomos) & 49 \\
\hline ¡Vale! (Moderna -4 tomos) & 27 \\
\hline Mucho (Moderna -3 tomos) & 8 \\
\hline Punto Final (Edelsa -1 tomo) & $13(\mathrm{v} .1)$ \\
\hline A Fondo (SGEL -1 tomo) & 114 \\
\hline Vamos a Hablar (Ática -4 tomos) & 80 \\
\hline
\end{tabular}

Notamos que los libros Punto Final y A Fondo, ambos clasificados como materiales para el nivel superior, son los que presentan más expresiones idiomáticas, respectivamente, 114 (ciento catorce) y 80 (ochenta). Por otra parte, el volumen 1 del manual Mucho, presenta el menor número de expresiones, o sea, 13 (trece). Cabe decir que los dos primeros manuales mencionados - Punto Final y A Fondo - son publicaciones españolas para alumnos extranjeros, no importando su lengua materna; ya el tercero - Mucho - está publicado en Brasil y se destina a alumnos de enseñanza básica y/o secundaria cuya lengua materna es el

${ }^{7}$ Aunque la elección haya sido al azar, establecimos algunos criterios generales: seleccionamos materiales conocidos y utilizados de manera más o menos generalizada en el país y con cierta difusión en la enseñanza fundamental y secundaria y cursos de idiomas. Además de eso, optamos por analizar manuales publicados tanto en España como en Brasil a lo largo de los años 90, basados en diversas propuestas metodológicas y clasificados, por sus autores, según el nivel al que se destinan.

${ }^{8}$ Aunque la serie está formada por tres volúmenes, sólo utilizamos en este estudio el tomo número uno. 
portugués. Los demás manuales son publicaciones brasileñas destinadas igualmente a la enseñanza básica y secundaria y son los siguientes: ;Vale! 27 (veintisiete expresiones en total), Vamos a Hablar 47 (cuarenta y siete expresiones en total) y Español sin Fronteras 49 (cuarenta y nueve expresiones en total). De lo expuesto, deducimos que:

a) hay un predominio de expresiones idiomáticas en los libros destinados a los niveles más avanzados de aprendizaje del idioma, lo cual parece indicar que en ese nivel las unidades fraseológicas figuran como un elemento de sofisticación lingüística;

b) los manuales de español de nivel avanzado publicados en España y analizados en este trabajo constituyen un único volumen en el cual han sido integradas las expresiones idiomáticas, curiosamente, en cantidad muy superior a las unidades fraseológicas presentes en las colecciones compuestas por más de un tomo;

c) en las colecciones brasileñas analizadas en su totalidad, Español sin Fronteras y Vamos a Hablar, no notamos una diferencia numérica representativa, ya que presentan, respectivamente, 49 y 47 expresiones idiomáticas. Sin embargo, sí hay una diferencia significativa entre los manuales mencionados y el ;Vale!, que contiene tan sólo 27 expresiones idiomáticas a lo largo de los cuatro tomos que componen la serie.

En un segundo momento tratamos de averiguar cómo figuraban tales expresiones en los libros, es decir, si se presentaban contextualizadas, si se incluían explicaciones, traducciones, ejercicios, etc. y constatamos la siguiente distribución?:

\begin{tabular}{|c|c|c|c|c|c|c|}
\hline Libro & $\begin{array}{c}\text { Ejercicios de } \\
\text { relacionar } \\
\text { columnas }\end{array}$ & $\begin{array}{c}\text { Cuadro con } \\
\text { equivalencias } \\
\text { en portugués }\end{array}$ & Glosario & $\begin{array}{c}\text { Textos } \\
\text { variados }\end{array}$ & $\begin{array}{c}\text { Ejercicios de } \\
\text { completar } \\
\text { huecos }\end{array}$ & Otros \\
\hline ESF & 07 & 14 & 05 & 18 & 04 & 01 \\
\hline $\mathrm{V}$ & 07 & - & - & 13 & - & 07 \\
\hline $\mathrm{M}$ & - & - & - & 11 & - & 02 \\
\hline $\mathrm{PF}$ & 02 & - & - & 30 & 02 & $\begin{array}{c}\text { Selección multiple: } 07 \\
\text { sustitución de términos: } 01 \\
\text { ordenación de términos: } 01 \\
\text { diálogos: } 02\end{array}$ \\
\hline $\mathrm{AF}$ & 08 & - & - & 28 & 25 & 19 \\
\hline $\mathrm{VH}$ & - & - & - & 35 & - & 12 \\
\hline
\end{tabular}

${ }^{9}$ A partir de este punto, nos valdremos del siguiente código para referirnos a cada una de las obras analizadas:

ESF - Español Sin Fronteras

V - ¡Vale!

M - Mucho

PF - Punto Final

AF - A Fondo

VH - Vamos a Hablar 
De lo anterior se infiere que existe una preocupación por parte de los autores de libros de texto no sólo en el sentido de incluir al menos algunas expresiones idiomáticas en los materiales, sino de contextualizarlas en textos de tipología variada (diálogos, narraciones, entrevistas, etc.) o bien de presentar sus equivalentes en portugués o el significado de la expresión. La explicación para la mayor incidencia de tales formas de tratamiento (presentación y/o práctica de las unidades fraseológicas) nos parecen claras, es decir, de acuerdo con lo ya expuesto, el significado no es fácilmente comprensible y esas unidades fraseológicas se actualizan en el discurso. De ahí que exista una preocupación mayor con esos dos aspectos.

Sin embargo, si se considera el aspecto cuantitativo, vemos que el número de expresiones idiomáticas incluidas en los materiales, incluso en aquellos de nivel superior, es muy reducido dada su gran utilización en la comunicación.

Además, aunque, como ya hemos señalado, haya una preocupación por aclarar el significado y por contextualizar este tipo de unidad fraseológica, no se puede afirmar que estemos frente a una metodología totalmente adecuada, ya que la pequeña cantidad de actividades propuestas difícilmente conducirá al uso espontáneo por parte de los alumnos. Notamos que no parece haber una preocupación por facilitar la apropiación de las expresiones idiomáticas pues no figuran propuestas concretas que traten de presentar y ejercitar las expresiones y, además, de permitir que se amplíe su uso en tareas prácticas que conduzcan a su posterior transposición a otros contextos.

Independientemente del local de publicación de los libros, se observa que no hay preocupación por incluir las expresiones en cuadros con equivalencias en portugués o incluso con su significado en español. Tampoco se ofrecen explicaciones relacionadas al sentido que las unidades fraseológicas adquieren en el discurso o sobre su uso. De idéntica manera, solamente una de las series analizadas incluye expresiones en el glosario que figura al final de cada volumen (Español sin Fronteras). Sin embargo, el número de expresiones ahí incluidas es insignificante con relación al total general presente en la obra.

Los ejercicios de tipo relacionar columnas aparecen en casi todos los libros analizados, lo cual puede indicar que se trata de una actividad que permite, de alguna manera, asociar las expresiones a su significado, sea a través de paráfrasis, sea a través de explicaciones sobre el sentido. Sin embargo, salvo en unas pocas excepciones, no se le ofrece al estudiante una aclaración previa que le facilite la comprensión del significado de cada una de las expresiones ahí incluidas, lo cual implica en la necesidad de orientación directa del profesor para que el aprendiz pueda realizar satisfactoriamente la actividad propuesta.

Algo semejante ocurre con los ejercicios en los que se debe completar un hueco con la expresión idiomática adecuada. Aunque no todos los libros analizados utilizan este tipo de actividad, los que la incluyen tampoco ofrecen elementos suficientes para que el alumno pueda lograr éxito sin la ayuda del profesor. También en este caso la comprensión efectiva del significado de cada una de las unidades fraseológicas se ve comprometida, puesto que no hay información anterior sobre los contextos de uso o sentidos que pueden adquirir según el contexto. 
Por fin, hay que señalar que, en ocasiones, las expresiones aparecen en actividades de carácter muy diverso, como ejercicios de elección múltiple, consignas de ejercicios, chistes, etc., lo cual no permite un análisis detallado sobre su validez o sobre los resultados que se pueden obtener. Por otra parte, muchas veces las expresiones idiomáticas no constituyen el objetivo de la actividad, sino que están insertadas en ejercicios cuyo propósito es la presentación o la fijación de determinados contenidos gramaticales (morfología, sintaxis, etc.). En estos casos, las unidades fraseológicas se mantienen al margen de la actividad, ya que sólo se pretende reforzar o sistematizar un aspecto lingüístico determinado, no directamente relacionado con las expresiones idiomáticas.

Una rápida mirada a unos cuantos ejemplos extraídos de los materiales analizados nos permitirá tener una visión más clara de lo que se acaba de exponer.

En la colección Español sin Fronteras se observa, claramente, que inexiste una preocupación específica con el significado y uso adecuado de las expresiones idiomáticas. Aunque se incluyen estas unidades fraseológicas a lo largo de los cuatro tomos que componen la serie, no se ofrecen ni los equivalentes más apropiados en portugués, ni explicaciones sobre el sentido que adquieren en diferentes contextos o sobre cómo emplearlas.

En el volumen 1, página 45, por ejemplo, se incluyen nueve expresiones idiomáticas ("asomar la cabeza", "andar con ojos", "quemarse las cejas", "tener la mano larga", "quedarse con un palmo de narices", "moderse los codos de hambre", "no tener pelos en la lengua", "dormir a pierna suelta" y "no dar pie con bola") y se pide a los estudiantes que asocien las expresiones populares con su "verdadero significado". Sin embargo, en ningún momento se le aclara al aprendiz o al profesor qué es una expresión idiomática y qué se entiende por "verdadero" (¿es que las expresiones idiomáticas poseen algún sentido que sea falso?) ni se presentan elementos que permitan realizar el ejercicio. Lo mismo ocurre en la página siguiente, cuando se repiten las mismas expresiones y se pide que las incluyan en nueve frases dadas por las autoras.

En los tomos 3 y 4 encontramos, además, otro tipo de problema. En estos casos las expresiones se incluyen en textos auténticos insertados en el apartado "Rincón de Lectura" que, según las autoras, visa a apresentar um pouco das culturas espanhola e hispanoamericana (pág.5, libro del profesor).

En la entrevista incluida en la página 112 del volumen 3 aparecen las expresiones "[estar] compuesta y sin novio", "(entrar) por la puerta grande" y "darse cuenta". Ya el texto El puercoespín mimoso, de Mario Benedetti, presente en la página 85 del volumen 4, incluye al menos quince expresiones idiomáticas. Sorprendentemente, no se incluye ningún tipo de explicación o actividad sobre el asunto, pese a que las autoras manifiesten, expresamente, que el apartado "Lectura" se preocupa con cuestiones culturales.

En síntesis, se puede afirmar que aunque en este material se incluyen algunas unidades fraseológicas, no se les da un tratamiento que permita inferir el significado de cada una de ellas ni cómo o cuándo usarlas de forma apropiada.

La serie ; Vale! restringe, más aún, el trabajo con las expresiones idiomáticas, tanto en lo que se refiere a la cantidad de ocurrencias ( 27 en total) como en la forma de presentarlas: ejercicios de relacionar columnas y en textos. En ambas modalidades de inclusión de las 
unidades fraseológicas no se ofrece, ni al profesor, ni al alumno, cualquier tipo de información sobre el significado o el uso.

Así, por ejemplo, en el tomo 1, página 83, en un texto elaborado con fines didácticos se incluyen las expresiones "estar chiflado" y "darse cuenta" pero no se retoman en los ejercicios. En el Glosario al final del volumen figura la palabra "chiflado" y la traducción louco pero en ningún momento se informa sobre el uso de la expresión, algo semejante a lo que ocurre en el volumen 2, página 10. En este caso, también en un texto didáctico, figura la expresión "en un santiamén" y en el Glosario sólo se ofrece la traducción em um instante, muito rápido, desconsiderando que existe una expresión equivalente en portugués (num piscar de olhos, num abrir e fechar de olhos).

En la página 72 del mismo volumen 2, en un texto auténtico consta la expresión "hacerse (algo) pesado". En esta ocasión tampoco se presentan aclaraciones de ningún tipo, ni siquiera en el Glosario. Es decir, además de que no se ofrecen ejercicios para que el alumno practique el uso de las expresiones ni se hace mención especial a ellas, tampoco hay preocupación en presentar equivalencias en la lengua materna de los estudiantes.

Ya en el texto Yo dos y tú uno de Antonio Rodríguez Almodóvar que figura en la página 37 del volumen 3 encontramos la expresión "dar el brazo a torcer" y, en este caso, tampoco se incluye ninguna traducción en el Glosario. Como en los volúmenes anteriores, la única referencia es la inclusión de la expresión en un texto y ninguna aclaración o ejercicio.

El mismo tipo de procedimiento se observa en el volúmen 4. Encontramos la expresión "cortar las alas" en uno de los chistes de la página 82; "estar en las nubes" y "[tener] mala pata" en un cuadro en la página 95 referido a formas para expresar interés, aprecio, simpatía, alegría, tristeza o decepción pero ninguna de ellas figura acompañada de explicaciones y tampoco se incluyen equivalentes en el Glosario.

Sin embargo, en las páginas 104 y 105 del mismo tomo 4 la situación se hace más grave. En el diálogo elaborado con fines pedagógicos hay huecos correspondientes a las expresiones "cara de viernes", "estar entre dos aguas" y "echar una mano" que el alumno deberá completar a partir de la audición de la grabación correspondiente. Ninguna explicación o traducción acompaña el texto. A continuación, se incluye un ejercicio de relacionar columnas: en la primera de ellas figuran quince expresiones idiomáticas con la palabra "cara" ("cara de pascua", "cara de viernes", "cara de acelga", "cara de ángel", etc.) y en la segunda, el sentido ("expresión alegre, placentera", "expresión triste", "persona de color pálido", "persona buena, a veces engañosa", respectivamente). La instrucción del ejercicio remite al texto anterior, pero no supone ningún tipo de ayuda para el estudiante:

¿Te diste cuenta de la expresión ‘cara de viernes’ en el diálogo? Según el sentido, relaciona las dos columnas. Tienes ahí muchas caras.

La pregunta que planteamos es ¿cómo los alumnos podrán inferir el significado si no se les ha ofrecido ninguna aclaración previa? Para el profesor tampoco resulta una actividad fácil pues la única sugerencia que se le presenta se refiere a una estrategia: 
Se puede destinar a cada alumno un tipo de cara y pedirles que elaboren frases y las digan al grupo. Se pueden aquí utilizar figuras existentes en revistas (fotos) u otra clase de material.

Pero si el profesor desconoce el sentido y el uso de alguna(s) de las expresiones incluídas en ese apartado, ¿cómo podrá llevar a cabo la actividad con éxito? Seguro que pondrá mala cara...

El libro Mucho (pág. 106, volumen 1), a su vez, incluye la expresión "soltar la rienda (a alguien)", que equivale, en portugués, a soltar as rédeas (de alguém). Esa expresión figura en un texto sobre horóscopo y va seguido de una actividad sobre el sentido de expresiones del texto. Las autoras dejan de mencionar el carácter idiomático de las expresiones, y específicamente la expresión que nos ocupa no se incluye en la actividad. Una nota le sugiere al profesor que dé a los alumnos el sentido de las expresiones.

Ya en la página 108 del mismo manual aparece la expresión "estar en la cresta de la ola", cuyo correspondiente en portugués es estar na crista da onda. Esa expresión figura también en una actividad sobre el horóscopo en la que se pide que el alumno la complete con informaciones sobre determinado signo, que él debe conocer previamente. En este caso, tampoco se ofrece ninguna información sobre la expresión idiomática, sea sobre su uso, sentido o equivalencia en portugués y la unidad fraseológica no forma parte de ninguna actividad específica relacionada a adquisición del léxico.

La expresión "decir cuántas son cinco", correspondiente, en portugués, a dizer com quantos paus se faz uma canoa, incluída en la página 135, aparece en el texto Ganarse la vida, de Jacinto Benavente. Dicho texto está insertado en el apartado "Lectura". Según las autoras del material, este apartado (que en la Guía del Profesor recibe el título "Textos literarios") tiene como objetivos "presentar lenguaje culto literario, estimular el análisis del lenguaje literario como instrumento de manifestación artística y orientar al alumno en la interpretación del mensaje del texto según la época y el contexto social del que proviene". (p.8). Dos preguntas de comprensión e interpretación acompañan el texto así como otras dos actividades. La segunda de ellas consiste en un ejercicio de relacionar columnas: en la primera se incluyen lo que las autoras denominan "expresiones" y en la segunda, "sentimientos". La tarea del alumno es relacionar cada expresión con un sentimiento. Entre las expresiones seleccionadas figura "decir cuántas son cinco", cuya correspondencia, en el ejercicio, es "amenaza". Nada más se dice sobre la expresión, ni siquiera se menciona tratarse de una expresión idiomática.

Como se observa, en este material no se enfatiza ni se demuestra interés por ningún trabajo específico con las expresiones idiomáticas, aunque se introduzcan a lo largo de sus páginas. El único modelo de actividad propuesto exige del profesor el conocimiento de las expresiones, pero no se le ofrecen subsidios para el caso de que él no lo posea.

En cuanto al manual Punto final, hay ejercicios de variada tipología como, por ejemplo, los de relacionar columnas, completar huecos, selección múltiple, reordenar términos y sustitución de términos. Además de esos, hay propuestas en las que las expresiones idiomáticas están presentes en textos pero no constituyen el objetivo del ejercicio. 
Como ejemplo del tipo de ejercicio de relacionar columnas escogemos el de número 5 de la página 114. En él se ofrece al alumno una columna con el significado y otra con las siguientes expresiones: 1. "ser la radio macuto" 2. "no tener parangón" 3. "marear la perdiz" 4. "estar de plantón" 5. "pintar la mona” y 6. "vigilar con colmillos de mastín”. Esas expresiones pertenecen a tres textos adaptados de Todos Vosotros, de M. Hidalgo y que se supone los alumnos ya los habrían leído. A pesar de la preocupación por ofrecer esas formas contextualizadas en los textos, no hay una propuesta de ampliación de su uso, de modo que el aprendiz pueda elaborar nuevos textos orales y/o escritos y automatizar su uso.

Un ejemplo de ejercicio de completar huecos es el de número 3 de la página 98 reproducido, parcialmente, a continuación:

3. Completa libremente estas frases que contienen modismos dándoles sentido completo.

- Estás en Babia, por lo tanto

- Estaba a la vista que

- No

porque no es para tanto.

estoy en blanco.

- Hoy estamos de suerte, por lo tanto

En el ejemplo anterior identificamos algunos problemas: a) hay una confusión terminológica, pues se consideran todas las expresiones como "modismos", lo que no es exactamente correcto y b) figuran expresiones idiomáticas y otras formas que no lo son, aunque sean, sin duda, de interés para la fraseología. Además, esos ejemplos pertenecen a la sesión "Aprieta los codos", dedicada al "repaso, consolidación y ampliación de estructuras de lengua de mayor rentabilidad o/y complejidad", según informan en el Prólogo los autores. Llama atención el hecho de que el objetivo sea el mencionado y que no figure ninguna propuesta que resulte en la consolidación del uso de las expresiones presentes en el ejercicio.

Otro tipo de ejercicio que merece la pena comentar es uno en el que se piden sustituciones. Se trata del ejercicio correspondiente a la letra "e" de la página 8 , reproducido, parcialmente, a continuación:

e. Sustituye los modismos en cursiva por expresiones normales.

- En estos momentos la situación está al rojo vivo.

- Sin saberlo dio en el blanco al tomar esa decisión.

- Desde que se le hundió el negocio, está sin blanca.

En el enunciado se emplea "modismos" por oposición a "expresiones normales" de lo que se deduce que las expresiones idiomáticas, de modo inadecuado, se consideran "modismos" y, por lo tanto, de cierta forma consistirían en una aberración ya que no son "normales". Además de eso, no hay una explicación acerca de los sentidos de modo a que el alumno sea capaz de utilizarlas en un contexto apropiado e incorporarlas a su discurso oral y/o escrito.

Teniendo en cuenta los ejemplos analizados constatamos que: 1. hay algunas impropiedades terminológicas en cuanto a las diferentes formas que figuran en los ejercicios, 
puesto que se consideran las expresiones idiomáticas como modismos; 2. no hay una definición y clasificación de las demás formas que no son propiamente expresiones idiomáticas; 3. los ejercicios, en general, atañen a la presentación de las expresiones y no hay una propuesta de memorización y de consolidación de su uso. En síntesis, no observamos un tratamiento adecuado, desde el punto de vista didáctico, para las expresiones idiomáticas en el material seleccionado, que le permita al aprendiz identificar, utilizar, automatizar y consolidar las formas propuestas en su discurso oral y/o escrito.

Como ya hemos señalado, el libro A Fondo, por ser un material pensado para un nivel superior y que contiene muchos textos extraídos de periódicos y revistas, incluye una cantidad mayor de expresiones idiomáticas y casi siempre aparecen primero contextualizadas y después en los ejercicios en los cuales el alumno comprueba el entendimiento y el uso adecuado. Eso es lo que ocurre, por ejemplo, en la página 49, cuando se presenta un texto publicado en el periódico El País semanal y en el cual figuran algunas expresiones que serán practicadas en los ejercicios de compresión, como se transcribe a continuación:

Intenta decidir por el contexto el significado de las palabras y expresiones coloquiales de la izquierda y relaciónalas con la columna de la derecha (algunas significan lo mismo).
a. pijo
1. Con mucha energía, volumen o fuerza.
b. chollo
2. Golpear.
c. aporrear
3. Golpes.
d. acabar como el rosario
4. cobrar mucho dinero a una persona
de la aurora por un objeto o servicio.
e. jalear
5. Canga, oportunidad.
f. sacar un ojo de la cara
6. Propio de los que dan mucha importancia a los signos externos de riqueza.
g. jarana
7. Jaleo, juerga.
h. a todo meter
8. Finalizar algo con un pelea o discusión.
i. porrazos
9. animar aplaudir.
j. mamporros

En la página 215, a su vez, aparecen en el texto Viva la televisión algunas expresiones y la propuesta de ejercicios es semejante, puesto que se pide que el alumno establezca la correspondencia entre todas las expresiones que figuran en el texto y su significado:

A cuál de las expresiones subrayadas en el texto corresponde cada una de estas definiciones?
a) Darse cuenta de la realidad
b) Mucho dinero
c) Tener cuidado con lo que se dice
d) Encontrar
e) Completamente
f) Lo imposible es imposible
g) Aumentar la calidad 
En la página 279 del mismo libro, hay otra propuesta de trabajo con las expresiones. Aquí el alumno tiene que intentar descubrir el significado de cada una de ellas y después lo comprobará en el ejercicio que sigue:

Intenta deducir el sentido de estas expresiones relacionadas en su origen con la vida en el campo. Las últimas son más difíciles, pero tu profesor te ayudará:

a. (Esta casa) está hecha una cuadra.

b. Hacer algo en menos que cante el gallo.

c. Ser más terco que una mula

d. (Esto) es/parece un gallinero.

e. Ser la oveja negra de la familia.

f. Acostarse con las gallinas.

g. (Eso) es harina de otro costal.

h. Pedir peras al olmo.

i. Volver al redil.

j. Cada muchuelo a su olivo.

k. No todo el monte es orégano.

1. Apear a alguien del burro / Apearse del burro.

m. Llevarse a alguien al huerto.

n. La cabra siempre tira al monte.

A continuación, el alumno deberá utilizar las expresiones para completar los huecos en las siguientes frases:

Completa las siguientes frases con las expresiones anteriores en su forma adecuada. No repitas ninguna.

a) Es un egoísta. Esperar que nos ayude sería

b) Lo llamamos y se presentó aquí . A mí no me hubiera dado tiempo ni de colgar el teléfono.

c) $\mathrm{Yo}$ A las nueve ya estoy en la cama.

d) No saldrás de casa hasta que recojas tu habitación; la tienes

e) Te dije que no te metieras por esta calle, que estaba prohibida, pero como

f) Yo soy Todos mis hermanos sacan buenas notas y yo apruebo por los pelos.

g) Después de diez años sin querer saber nada de su familia, decidió

h) Está equivocado pero no hay manera de

i) ¡Se acabó la fiesta! ;

j) Tú te has creído que montar un negocio es llegar y empezar ganar dinero, pero

Claro que si me dices que el dinero es lo de menos y lo que te gusta es el riesgo, eso ya

k) No sé si fiarme en el, parece que ha cambiado y que está más formal, pero

1) ¿Os podéis callar? Esto

m) Yo no me fío de éste. Creo que está intentando

Las propuestas de ejercicios presentadas por los autores del libro A Fondo también reflejan el tratamiento que se les da a las expresiones idiomáticas. Observamos que estas unidades fraseológicas aparecen mezcladas con otras construcciones propias de la lengua española como jergas, giros, refranes, locuciones, dichos o palabras que adquieren significados específicos según la situación o el contexto social en que se utilizan. $\mathrm{Su}$ presentación se da a partir de un texto auténtico con el propósito de poner el alumno en contacto con la situación. Este tipo de estrategia se justifica pues con ese procedimiento se 
intenta despertar el interés y la curiosidad del alumno que trata de entender el texto de forma global y no de conocer, en este contacto inicial, específicamente el significado de cada expresión; eso será explicitado en los ejercicios posteriores. Como no se trata de un material específico para la enseñanza de español a brasileños no hay explicaciones, traducciones, comparaciones, equivalencias o cualquier intento de hacer una aproximación a la realidad brasileña. La tipología de ejercicios tampoco presenta novedades: las actividades incluidas se restringen a completar huecos, sustituir frases por palabras o expresiones y relacionar columnas.

Una primera mirada a los libros de la colección Vamos a hablar, de Editora Ática, deja claro que aunque el material presente algunas expresiones idiomáticas en los textos y ejercicios (sobretodo en los volúmenes 3 y 4), no les ofrece a los alumnos un trabajo específico que ayude a comprenderlas y usarlas con adecuación.

En la página 76 del libro 2, por ejemplo, la expresión "no tener ni pies ni cabeza" aparece en el diálogo que abre la unidad. Tras ese diálogo se incluyen preguntas de comprensión del texto y, a seguir, un apartado que lleva por título "Giros y expresiones" en el cual figura dicha expresión. Sin embargo, no se le ofrece al alumno ni el significado ni otros ejemplos de uso de la expresión que permitan inferir el sentido. Hay que añadir que en ese apartado se mezclan expresiones idiomáticas, refranes, locuciones y otras expresiones fijas como “¿De parte de quién?, ¡Que tengas suerte”, ¡Lo que me faltaba”, ¡Dios Santo”, ¡Cuánto lo siento!.

De esa misma manera se muestran otras 26 expresiones, lo que representa más de la mitad de todas las expresiones idiomáticas presentes en la colección. Las demás se incluyen en frases o en textos de ejercicios pero no se hace ninguna referencia especial a ellas:

2) Complete [con los verbos entre paréntesis conjugados].

... Ramón _____________ (conducir) la reunión de forma lamentable. (conseguir). Al fin nos (parecer) que (querer) irritarnos. Y lo la aurora.

todos y la sesión (acabar) como el rosario de la aurora.

[Volumen 4, página 53]

1) Ponga en pretérito perfecto simple las siguientes frases.

Ej.: Duermo a pierna suelta.

Ayer dormi a pierna suelta.

[Volumen 2, página 49]

En algunos casos se incluye la equivalencia en portugués de una expresión idiomática en el libro del profesor; sin embargo, no se le ofrece al docente ningún ejemplo más. Hay que considerar, también, que los contextos en los que figuran las expresiones no constituyen fuentes auténticas, sino que son especialmente elaborados para fines didácticos, lo que indica no haber una preocupación con la enseñanza de su uso efectivo a partir de su contextualización en textos reales. 
En síntesis, podemos afirmar que no siempre la inclusión de expresiones idiomáticas en los libros seleccionados se hace de manera eficaz, puesto que, en ocasiones, sea la forma de presentarlas, sea la forma de practicarlas, no permite que el alumno se apropie del significado ni que lo incorpore a su vocabulario activo.

\section{A MODO DE CONCLUSIÓN}

A lo largo de este estudio expusimos nuestra reflexión acerca de la importancia de la enseñanza del léxico y de las dificultades en este proceso. Hicimos hincapié en la necesidad de valorar la enseñanza del léxico como fundamental para lograr una competencia comunicativa en la lengua meta, acorde al nivel de los estudiantes y de los objetivos del curso. Además, nos ocupamos de algunos aspectos que estimamos importantes como, por ejemplo, la selección del léxico y el modo de enseñarlo. En cuanto al primer punto, tratamos de la necesidad de seleccionar el léxico sea como hablantes de una lengua, sea como aprendices y profesores, y aun, de algunos criterios establecidos para dicha selección. En cuanto al segundo aspecto, no menos importante, enfatizamos las unidades fraseológicas, como objeto de enseñanza y, en especial, mencionamos las expresiones idiomáticas, objeto de nuestra actual investigación. Señalamos también algunas propuestas para la enseñanza de las unidades fraseológicas, a partir de las aportaciones de Penadés Martínez (1999) y de nuestra experiencia docente.

Nuestro objetivo en este estudio fue el de reflexionar acerca del léxico e incluir en el centro de nuestras preocupaciones la enseñanza de las unidades fraseológicas, más específicamente, las expresiones idiomáticas. Ese interés se justifica debido al reducido número de investigaciones sobre el asunto en Brasil, a los problemas que tenemos con nuestros alumnos que desean conocer más de esas expresiones y lograr una competencia comunicativa más amplia. Además, añadimos a esas razones, la necesidad de producir materiales adecuados para la enseñanza de las expresiones idiomáticas a brasileños que puedan suplir, en parte, las mencionadas carencias y que de alguna manera la teoría sirva de apoyo a la aplicación y a la práctica docente.

Esperamos que con este breve estudio podamos contribuir para que haya un cambio en ese panorama y profundizar nuestro proyecto de investigación con la finalidad de echar algunas luces sobre la enseñanza del léxico, en el caso, la enseñanza de las unidades fraseológicas a brasileños.

\section{REFERENCIAS BIBLIOGRÁFICAS}

ALVES, A.N.M. y ALVES, A.M. (1999). ;Vale! Español para brasileños. São Paulo, Moderna. (2001). Mucho. Español para brasileños. São Paulo: Moderna. (v.1)

BELTRÁN, M.J. y YÁÑEZ TORTOSA. (1999). Modismos en su salsa. Modismos, locuciones y expresiones fijas en sus contextos. Madrid: Arco/Libros. 
CÁCERES, M.R, JIMÉNEZ, F.P. et alii. (1997). Vamos a hablar. São Paulo: Ática.

CORONAdo GONZÁleZ, M.L. et alii. (1994). A Fondo. Curso Superior de Español para Extranjeros. Lengua y Civilización. Madrid, SGEL.

EVEREST Diccionario Práctico de locuciones y frases hechas. (1998). León: Evergráficas.

GARCÍA, M.A.J. y HERNÁNDEZ, J.S. (1997). Español sin fronteras. São Paulo: Scipione.

HALLIDAY, M.A.K. et alii. (1974). As ciências lingüísticas e o ensino de línguas. Petrópolis: Vozes.

JIMÉNEZ, A.B. (1997). Diccionario Espasa. Dichos y frases hechas. Madrid: Espasa.

LIMA, L.M. (1997). La traducción de los modismos en la enseñanza de ELE, en: Anuario Brasileño de Estudios Hispánicos, 7: p. 63-68. São Paulo: Embajada de España en Brasil/Consejería de Educación y Ciencia.

LLOBERA, M. et alii. (1995). Competencia comunicativa. Documentos básicos en la enseñanza de lenguas extranjeras. Madrid: Edelsa.

MARCOS DE LA LOSA, M.C. \& OBRA ROdRÍGUEZ, M.R. (1997) Punto Final. Curso Superior E.L.E. Madrid: Edelsa.

ORTIZ ÁLVAREZ, M.L. (1997). As expressões idiomáticas dentro da obra lexicográfica, en: Revista Brasileira de Lingüística, 9/1. São Paulo: Plêiade.

PASSEL, F.V. (1983). Ensino de línguas para adultos. São Paulo: Pioneira.

PENADÉS MARTÍNEZ, I. (1999). La enseñanza de las unidades fraseológicas. Madrid: Arco/Libros.

ROMERO GARCÍA, C. (1998). Cómo molar cantidubi: expresiones para enterarse de qué va la fiesta, en: Boletín de ASELE, mayo/1998, Santiago de Compostela.

SLAGTER, P.J. (1988). ¿Qué palabras que enseñar?, en: Revista CABLE, Madrid: Equipo CABLE.

TAGNIN, S.O. (1989). Expressões idiomáticas e convencionais. São Paulo: Ática.

ZULUAGA, A. (1980). Análisis y traducción de unidades fraseológicas desautomatizadas, en: Introducción al estudio de las expresiones fijas. Frankfurt am Main: Peter D.Lang.

http://www.centroatl.pt/espacidiomatico.faq.html 